\title{
Progressive intramyocardial dissecting hematoma in the apex of the left ventricle after percutaneous coronary intervention
}

\author{
Ying Wei, Bo Zhang \\ Department of Ultrasound in Medicine, Shanghai East Hospital, Tongji University School of Medicine, Shanghai, China \\ Correspondence to: Bo Zhang. Department of Ultrasound in Medicine, Shanghai East Hospital, Tongji University School of Medicine, Shanghai \\ 200120, China. Email: zhangbodongfang@qq.com.
}

Submitted Mar 29, 2020. Accepted for publication Jul 27, 2020.

doi: 10.21037/qims-20-508

View this article at: http://dx.doi.org/10.21037/qims-20-508

\section{Introduction}

Intramyocardial dissecting hematoma (IDH) is considered a type of incomplete cardiac rupture, and its clinical symptoms may vary from asymptomatic remission to cardiac death. It occurs mostly after myocardial infarction (MI), percutaneous coronary intervention (PCI), coronary artery bypass surgery, or cardiac surgery. It is a rare complication with a high fatality. Persistent ST-elevation is important in determining suspected IDH, which is confirmed by its unique echocardiography, while serial echocardiography is useful and may guide outcomes (1). In our case, echocardiography was found to be effective in the diagnosis and monitoring of $\mathrm{IDH}$, and we were able to choose the best treatment strategy for the patient as a result. The present study was conducted in accordance with the Quantitative Imaging in Medicine and Surgery (QIMS) guideline checklist.

\section{Case presentation}

A 59-year-old man with a history of smoking was admitted to our medical center for intermittent chest pain. An urgent electrocardiogram showed acute MI with ST segment elevation on the II, III, avF, I, avL, and V2-V6 leads. Transthoracic echocardiographic examination revealed an ejection fraction (EF) of about $45 \%$, with an aneurysm akinetic without thrombus at the apical segments of the left ventricle, accompanied by mild regurgitation of the mitral valve and medium regurgitation of the tricuspid valve with minimal pericardial effusion (Figure 1A). Creatine kinase isoenzyme-MB (CK-MB), cardiac troponin I (TNI), and pro-brain neuropeptide (pro-BNP) were found to be beyond normal levels (CK-MB: $296.4 \mathrm{ng} / \mathrm{mL}$; TNI: $3.03 \mathrm{ng} / \mathrm{mL}$; pro-BNP: 3,859 ng/L). Coronary angiography revealed that the anterior descending artery (LAD) was partially occluded by the thrombus, whereas the left circumflex and right coronary arteries were normal. Intravascular stenting was performed in the $\mathrm{LAD}$, and there were no complications during the procedure. The patient complained of chest discomfort after PCI. He had acute left heart failure several times, but echocardiography showed no significant changes compared to preprocedure. The followup electrocardiogram showed ST-elevation.

The patient complained of severe chest pain on PCI day 11 , and echocardiography discovered thrombosis in the left ventricle apex aneurysm; a hematoma was located between the myocardial fibers layers of the aneurysm (Figure 1B). There was blood flow in and out of the hematoma during systole and diastole (Figure 1C,D). The size of the hematoma varied with the cardiac cycle. Perforation occurred due to the aneurysm and the left ventricular cavity. Echocardiography showed that the epicardium seemed to be intact. We suspected that the hematoma was an IDH. However, cardiac magnetic resonance imaging (MRI), not echocardiography, is the gold standard for the diagnosis of IDH. MRI was suspended for his recurrent acute heart failure, and the diagnosis of IDH could not be confirmed. Conservative therapy was therefore commenced, and echocardiogram was used to carefully monitor the progression of the hematoma. When the patient's heart failure symptoms had almost stopped, MRI showed an apical hematoma at the area of the ruptured orifice covered by the intact epicardium (Figure 1E), and 

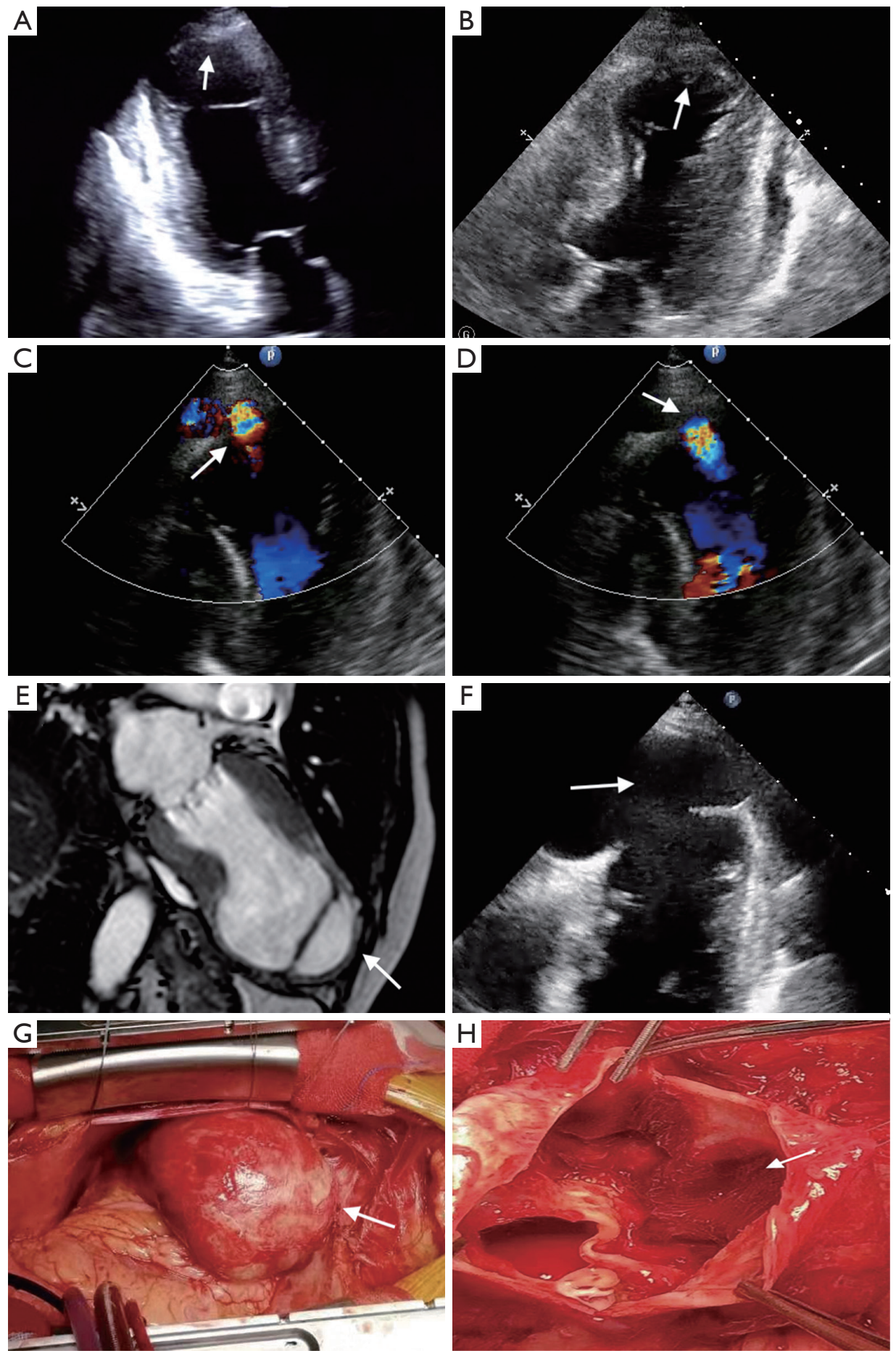

Figure 1 Echocardiography showed no thrombus at the apex (white arrow) of the left ventricular (A). Apical five-chamber view revealed thrombosis (white arrow) in the left ventricle apex aneurysm (B). Hematoma located between the myocardial fibers of the aneurysm. Size of the hematoma varied in the cardiac cycle. Blood flow into the hematoma (white arrow) in the systole period (C) and out of the hematoma (white arrow) in the diastole period (D). Hematoma was covered by an intact epicardium (white arrow), as found in magnetic resonance imaging (E). Size of the hematoma and the orifice had increased (white arrow) (F). Large epicardium of the hematoma was unbroken (white arrow) $(\mathrm{G})$. Endocardium and muscle layer structure ruptured, and the orifice and some thrombi were evident (white arrow) (H). 


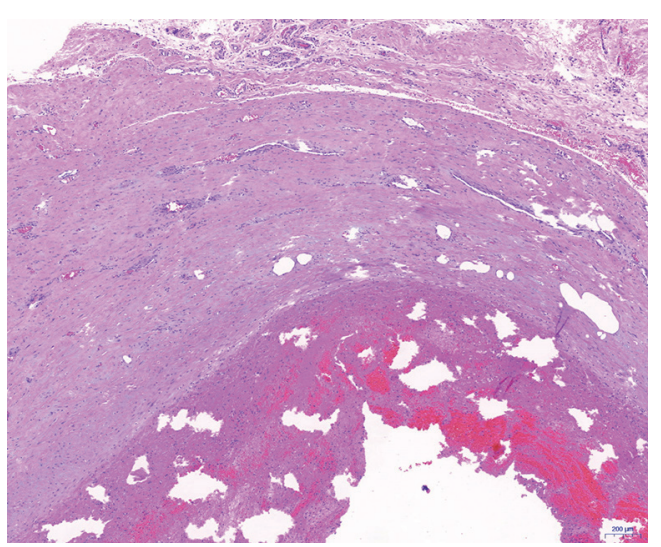

Figure 2 Histological examination showed that the epicardial layer was fibrous tissue, and mixed thrombi were attached to the inner wall of the epicardium.

the diagnosis of IDH was confirmed. The size of the IDH and the orifice was found to have increased (Figure 1F). To prevent epicardial rupture or sudden death, aneurysmectomy was performed. A large hematoma was exposed at the apex of the left ventricle, and the epicardium was unbroken (Figure 1G). The endocardium and muscle layer structure ruptured, and the orifice and some thrombi in the IDH were evident (Figure 1H). Pathological examination showed that the muscle layer structure ruptured, and the epicardial layer maintained its integrity with fibrous tissue; mixed thrombi, dominated by red thrombi, were attached to the inner wall of the epicardium (Figure 2). The ventricular aneurysm was resected, and left ventricular remodeling was performed. At follow up, echocardiography was performed, and there was no IDH recurrence. The patient recovered and was discharged from hospital 10 days after the surgery. Follow-up echocardiography of the patient 2 months after discharge revealed improvement in $\mathrm{EF}$ and no thrombus in the apex.

\section{Discussion}

IDH is considered a type of incomplete cardiac rupture and has a high mortality. It occurs in the myocardial layer between the endocardium and epicardium. During echocardiography, the appearance of a neocavitation with an echo lucid center in the infarcted myocardium communicating with the ventricular cavity is indicative of an IDH. As it has an intact epicardium, IDH are also known as subepicardial aneurysms, pseudo-false aneurysms, or pseudo-pseudoaneurysms (2-5). IDH mainly occur after
MI, PCI, coronary artery bypass surgery, or cardiac surgery. Intramuscular bleeding may be caused by the intramuscular vascular fragility, a decrease in tensile strength, or an increase in intravascular pressure in the infarcted tissue (6). It could further expanded into the dissection tract and rupture adjacent structures, or can spontaneously resolve (7). Some cases can evolve into full-blown cardiac rupture. The clinical symptoms of IDH may vary from asymptomatic remission to cardiac death. Due to its rarity and atypical clinical presentation, IDH is often misdiagnosed or missed. The endocardium shows discontinuity at the neck of the aneurysm, and often epicardial membrane integrity cannot be confirmed by echocardiography. IDH is sometimes missed due to the limited requirement for expert observers and the low quality of images. MRI is necessary to accurately diagnose IDH. Transthoracic echocardiography is considered to be the non-invasive technique of choice for diagnosing IDH at the bedside. In patients with MI who do not have obvious pain relief after PCI, and for whom electrocardiogram indicates persistent ST-elevation accompanied by slight or mild of pericardial effusion, the possibility of IDH should be considered. Therefore, it is important to carefully monitor and follow up patients in case of suspected IDH. In our case, we considered a diagnosis of IDH for our patient following echocardiography. We found, by cardiac ultrasound, that the hematoma continued to gradually increase in size, which was later confirmed by MRI. Echocardiography was found to be effective in the diagnosis and monitoring of the IDH.

There is disagreement about how best to treat IDH. Some believe that cardiac surgery therapy should be immediately performed, whereas others suggest that conservative management is successful in asymptomatic chronic IDH (8). The decision to adopt a surgical or conservative strategy mainly depends on the clinical and hemodynamic stability of the patient. The precise and temporal assessment of the structure of the hematoma is important. Spinelli et al. studied a series of conservatively treated patients with IDH in the setting of anterior STelevation MI, and found that IDH was associated with poor angiographic outcomes, severe impairment of left ventricular function, adverse left ventricular remodeling, and worse long-term prognosis (9).

We performed different therapies according to the growth of the hematoma in a different period. Echocardiography is an effective, non-invasive monitoring tool; however, surgery should be the first choice if the hematoma has grown within a short period of time 
according to echocardiographic examination. Surgery should be guided by imaging for the best patient outcomes.

\section{Acknowledgments}

Funding: The present study was supported by the Important Weak Subject Construction Project of Pudong Health Bureau of Shanghai (no. PWZbr 2017-09). The authors report grants from that Important Weak Subject Construction Project of Pudong Health Bureau of Shanghai for the conduct of the study.

\section{Footnote}

Conflicts of Interest: Both authors have completed the ICMJE uniform disclosure form (available at http:// dx.doi.org/10.21037/qims-20-508). BZ reports grants from Important Weak Subject Construction Project of Pudong Health Bureau of Shanghai, during the conduct of the study; YW reports grants from Important Weak Subject Construction Project of Pudong Health Bureau of Shanghai, during the conduct of the study.

Ethical Statement: Written informed consent was obtained from the patient for publication of this study and any accompanying images. A copy of the written consent is available for review by the editor-in-chief of this journal.

Open Access Statement: This is an Open Access article distributed in accordance with the Creative Commons Attribution-NonCommercial-NoDerivs 4.0 International License (CC BY-NC-ND 4.0), which permits the noncommercial replication and distribution of the article with the strict proviso that no changes or edits are made and the original work is properly cited (including links to both the formal publication through the relevant DOI and the license). See: https://creativecommons.org/licenses/by-nc-nd/4.0/.

\section{References}

1. Vargas-Barrón J, Romero-Cárdenas A, Roldán FJ, Molina-
Carrión M, Avila-Casado C, Villavicencio R, MartínezSánchez C, Lupi-Herrera E, Zabalgoitia M. Longterm follow-up of intramyocardial dissecting hematomas complicating acute myocardial infarction. J Am Soc Echocardiogr 2005;18:1422.

2. Epstein JI, Hutchins GM. Subepicardial aneurysms: a rare complication of myocardial infarction. Am J Med 1983;75:639-44.

3. Zheng Y, Sun JP, Shen X, Qiao Z, Ge H, Li Z, He B, Yu, CM. Subepicardial Aneurysm That Was Diagnosed by Cardiac Imaging and Underwent Successful Surgery. Circulation 2015;132:e149-51.

4. Inoue T, Hashimoto K, Bando K, Yoshitake M. Left ventricular pseudo-false aneurysm perforating into the right ventricle. Interact Cardiovasc Thorac Surg 2015;21:137-9.

5. Gollol-Raju N, Olearczyk B, Johnson R, Menzies DJ. Pseudo-pseudoaneurysm: a rare and unexplored mechanical complication of myocardial infarction. J Am Soc Echocardiogr 2007;20:1317.e1-3.

6. Vargas-Barrón J, Roldán FJ, Romero-Cárdenas A, MolinaCarrión M, Vázquez-Antona CA, Zabalgoitia M, Martínez Rios MA, Pérez JE. Dissecting intramyocardial hematoma: clinical presentation, pathophysiology, outcomes and delineation by echocardiography. Echocardiography 2009;26:254-61.

7. Hajsadeghi S, Amirfarhangi A, Pakbaz M, Pazoki M, Tanha K. Postinfarction intramyocardial dissection, an interesting case report and systematic review. Echocardiography 2020;37:124-31.

8. Pontone G, Bertella E, Andreini D, Pepi M, Polvani G. Postoperative dissecting ventricular haematoma: a conservative strategy with a cardiac magnetic resonance imaging follow-up. Eur Heart J Cardiovasc Imaging 2014;15:1151.

9. Spinelli L, Stabile E, Giugliano G, Morisco C, Giudice CA, Imbriaco M, Santoro M, Esposito G, Trimarco B. Intramyocardial dissecting hematoma in anterior wall ST elevation myocardial infarction: impact on left ventricular remodeling and prognosis. Int J Cardiovasc Imaging 2018;34:201-10.
Cite this article as: Wei Y, Zhang B. Progressive intramyocardial dissecting hematoma in the apex of the left ventricle after percutaneous coronary intervention. Quant Imaging Med Surg 2020;10(12):2370-2373. doi: 10.21037/ qims-20-508 